\section{PMO-213 A PROSPECTIVE, RANDOMISED STUDY OF DOUBLE- BALLOON COLONOSCOPY VS CONVENTIONAL COLONOSCOPY FOR TECHNICALLY DIFFICULT COLONOSCOPY}

doi:10.1136/gutjnl-2012-302514b.213

E J Despott, ${ }^{*}$ A Murino, L A Bourikas, M Nakamura, C Fraser. Wolfson Unit for Endoscopy, St Mark's Hospital and Academic Institute, Imperial College London, London, UK

Introduction Technically "difficult" (TD) colonoscopy may lead to incomplete colonoscopy, increased patient discomfort and potentially higher sedation dose. Parameters which are associated with TD colonoscopy include female gender, age, BMI, history of major pelvic/abdominal surgery or chronic constipation and previous failed conventional colonoscopy (CC). Double-balloon colonoscopy (DBC) may facilitate TD colonoscopy.

Methods We performed a prospective, randomised study comparing DBC and CC for TD colonoscopy. Patients referred for a colonoscopy were screened for parameters predictive of TD colonoscopy using a scoring system developed at our institution. Only patients with scores $\geq 3$ qualified for recruitment; patients were then randomised to DBC or CC, performed by 1 of 2 designated experienced endoscopists. Collected data included patient pain/discomfort, difficulty of colonoscopy as judged by the endoscopist, sedation dose, colonoscopy completeness, time taken for caecal intubation/ procedure completion and recovery time. On recovery, patients were asked to rate their satisfaction and whether they would opt to undergo the same type of colonoscopy in future.

Results Forty-four patients were recruited (DBC, $n=22$; CC, $n=22$ ). Median calculated pre-procedure difficulty scores were the same for both groups (4.0 vs 4.0, $\mathrm{p}=0.16$ ). Mean patient discomfort and pain scores were significantly lower for the DBC group ( 2.6 vs $4.8, p=0.004$ and 2.4 vs $4.9, p=0.002$, respectively). Median doses of midazolam and pethidine used were significantly lower for DBC procedures (0 vs $1.25 \mathrm{mg}, \mathrm{p}=0.023$ and 0 vs $25 \mathrm{mg}, \mathrm{p}=0.014$, respectively). While differences in mean times taken for caecal intubation at DBC vs CC were similar ( 17.5 vs 14 min, $\mathrm{p}=0.18$ ), DBC facilitated total colonoscopy in all 22 cases whereas 6 CC procedures were only completed with the aid of a magnetic endoscopic imager (MEI), required after a mean of $15 \mathrm{~min}$ of failing to progress. Another $3 \mathrm{CC}$ cases failed to achieve caecal intubation despite use of a MEI and even a paediatric colonoscope. Median recovery time was significantly shorter for DBC (5 vs $20 \mathrm{~min}, \mathrm{p}=0.014$ ). Endoscopists found $\mathrm{DBC}$ to be significantly easier to perform than CC (median difficulty VAS: 3.6 vs 6.6 $\mathrm{p}=0.0005$ ) and significantly more patients in the DBC group were satisfied (DBC vs CC median Likert satisfaction score: 5.0 vs 3.0, $\mathrm{p}=0.006$ ). All patients in the DBC group said they would have DBC again but $41 \%$ of patients in the CC group said they would consider an alternative procedure instead.

Conclusion Our study suggests that DBC is a more comfortable and easier alternative to CC for TD cases. Since it appears to require less use of sedation, recovery also appears to be faster, with higher patient satisfaction levels.

Competing interests E J Despott: Grant/Research Support from: Fujifilm \& Imotech Medical (UK), A Murino: Grant/Research Support from: Fujifilm \& Imotech Medical (UK), L Bourikas: None declared, M Nakamura: None declared, C Fraser: None declared.

\section{PMO-214 DOUBLE BALLOON COLONOSCOPY: SINGLE CENTRE EXPERIENCE IN PATIENTS WITH PREVIOUSLY FAILED COLONOSCOPY}

doi:10.1136/gutjnl-2012-302514b.214

E G Alexandridis, ${ }^{*}$ A Koulaouzidis, K Trimble, J N Plevris. Centre for Liver and Digestive Disorders, Royal Infirmary of Edinburgh, Edinburgh, UK

Introduction Double balloon endoscopy is considered a technique for the investigation of the small-bowel. Recently, Double balloon colonoscopy (DBC) using dedicated colonoscopes has been shown to be associated with very high rates of caecal intubation. In this study we report the $\mathrm{DBC}$ experience in our centre as second line endoscopic investigation in patients who failed conventional colonoscopy.

Methods Retrospective review of patients referred for DBC to our Centre from July 2009 to January 2012.

Results Twenty-three consecutive patients (12 male/11 female mean age $60.2 \pm 16$ years) underwent DBC. The sedation used was midazolam/fentanyl (mean: 3.5/75 mg). 19/23 had a DBC due to previously failed colonoscopies. In four, DBC was first choice test as they had unpleasant experiences from conventional colonoscopy in the past. In $7 / 23(30.4 \%)$, there was evidence of previous abdominal surgery. In $4 / 23(17.4 \%)$ a fixed and/or acutely angulated sigmoid was the cause of failure. In the remaining 12 patients, significant patient discomfort was the principal cause of failure. Nine patients (39.1\%) had one failed colonoscopy, six (26.1\%) had two failed colonoscopies, while four $(17.4 \%)$ had more than two failed colonoscopies. In 22/23 DBC (95.7\%) was successful; the entire colon and terminal ileum DBC were examined in all cases. No immediate or delayed complications were recorded. Patient tolerability was very good.

Conclusion $\mathrm{DBC}$ is a safe and reliable method for complete colon examination and it is an important alternative technique in cases where a conventional colonoscopy has failed. Patient groups that are more likely to benefit are those with adhesions due abdominal surgery or fixed and angulated sigmoid colon. The technique is currently limited to few centers only, but the success rate and the very good patient tolerability suggest that it should be considered as an alternative in challenging cases.

Competing interests E. Alexandridis Grant/Research Support from: Fujifim, Imotech, A. Koulaouzidis: None Declared, K. Trimble: None Declared, J. Plevris Grant/Research Support from: Fujinon, Imotech.

\section{PM0-215 MOVIPREP VS PICOLAX FOR SCREENING COLONOSCOPY}

doi:10.1136/gutjnl-2012-302514b.215

E Howells. ${ }^{*}$ Bowel Screening Wales, Public Health Wales, Llantrisant, UK

Introduction This study involves a retrospective review of the standards of bowel preparation during screening colonoscopy at a Local Assessment Centre (LAC) in Wales. The audit data were collected over a 12-month period with an aim to identify which preparation allowed for optimum visualisation of the bowel during screening colonoscopy.

Methods The data on 224 participants who had colonoscopy performed through the Bowel Screening Wales (BSW) programme at a LAC between January 2009 and January 2010 were analysed. Inclusion criteria were that the participant must have been prescribed either Picolax or Moviprep and that they had a complete colonoscopy with examination to the Caecum. Two hundred and five participants were included, 144 participants were prescribed Picolax and 61 participants were prescribed Moviprep. Nineteen participants were excluded from the audit because they either had a limited procedure such as flexible sigmoidoscopy, or were prescribed an alternative bowel preparation. To ensure robust statistical analysis data were obtained from both Screening Colonoscopist and Specialist Screening Practitioner reports. Bowel preparation was scored according to visual appearance during the colonoscopy procedure. The categories were classified into:

- Good

- Adequate

- Unsatisfactory 